\title{
Detection of Crystalline Components in Amorphous Solid Dispersions by Correlative Imaging Techniques
}

\author{
Joseph Neilly ${ }^{1}$ and John Roth ${ }^{1}$ \\ 1. AbbVie Inc. NCE Analytical Chemistry, North Chicago, IL
}

Poorly soluble pharmaceutical compounds generally have low bioavailability. Improving solubility of these compounds can improve bioavailability, and one approach to improve solubility is to formulate crystalline drugs in an amorphous state. Amorphous compounds are kinetically more soluble but also thermodynamically less stable than the crystalline form. Various techniques have been employed to stabilize amorphous solids primarily by dispersing them in a variety of stabilizing materials [1].

Since crystallization of an active pharmaceutical ingredient (API) in amorphous solid dispersions (ASD) could have detrimental effect on the quality of the product, detecting and monitoring crystallization is extremely important to monitoring the stability of the product. Numerous spectrographic and imaging techniques for assessing ASDs have been compared by these authors [2]. Continuing with this work, imaging by polarized light microscopy (PLM), scanning electron microscopy (SEM), elemental mapping by energy dispersive x-ray spectroscopy (EDS), and x-ray microscopy (XRM) were compared for their ability to detect crystals in powder blends spiked with crystalline active pharmaceutical ingredient (API) and a model ASD system with residual crystals.

For this work, the model ASD was developed by dissolving acetaminophen (APAP) in copovidone via a hot melt extrusion process. The APAP is mixed with copovidone at elevated temperatures to form a solid solution, extruded through a die opening, cooled and milled to a powder. Extrusion conditions were varied to produce samples with varying levels of undissolved crystalline APAP. [3] A portion of the powder was imaged directly and some was pressed into tablets and examined. All of the above imaging techniques were used to measure the relative levels of crystallinity and show the distribution of the crystals in the system.

PLM easily detected birefringent crystalline material in thin transparent powders (Figure 1). However, the samples must be transparent for this method to work, thus PLM is not suitable for intact tablets. Previous work has shown that SEM/EDS can visualize spiked API in an amorphous matrix where sulphur was a unique element to the API [4]. This approach is well suited for imaging crystals on surfaces regardless of sample thickness or transparency. However in the APAP based model, SEM/EDS could not detect the APAP (Figure 2). This is likely due to the lack of a unique hetero atom in the APAP compared to the copovidone.

J.D. Ferrara et al recently used X-ray microscopes to image materials in pharmaceutical compounds [5]. By tuning the energy of the x-ray source they were able to optimize contrast for low atomic number materials. New developments in XRM shows promise for imaging crystals in ASD systems since it has the ability to image optically opaque or thick samples and will be investigated with the APAP based model system. [3] 


\section{References:}

[1] G Zografi in "Pharmaceutical Amorphous Solid Dispersions", ed A Newman, (John Wiley \& Sons, Hoboken, NJ) pp. 22-38.

[2] A. Vogt and J. Neilly, Microsc. Microanal. 21, Suppl 3 (2015) pp. 549-550

[3] The authors acknowledge sample preparation by A Ruggles, Formulation Sciences, Abbvie Inc.

[4] J Neilly \& J Roth, Microsc. Microanal. 21, Suppl 3 (2015) pp. 551-552

[5] J Ferrara et al, Microsc. Microanal. 20, Suppl 3 (2014) pp. 674-675
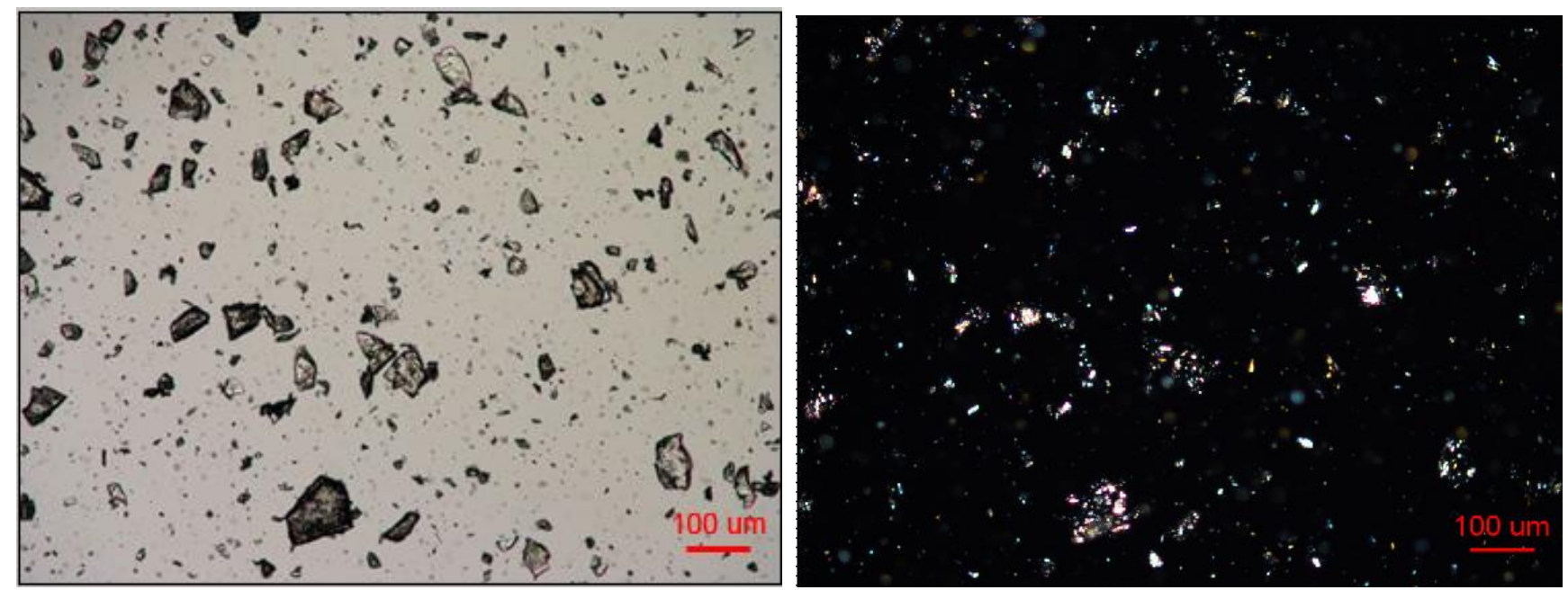

Figure 1. Plane (left) and cross polarized (right) images of ASD with embedded crystalline material.
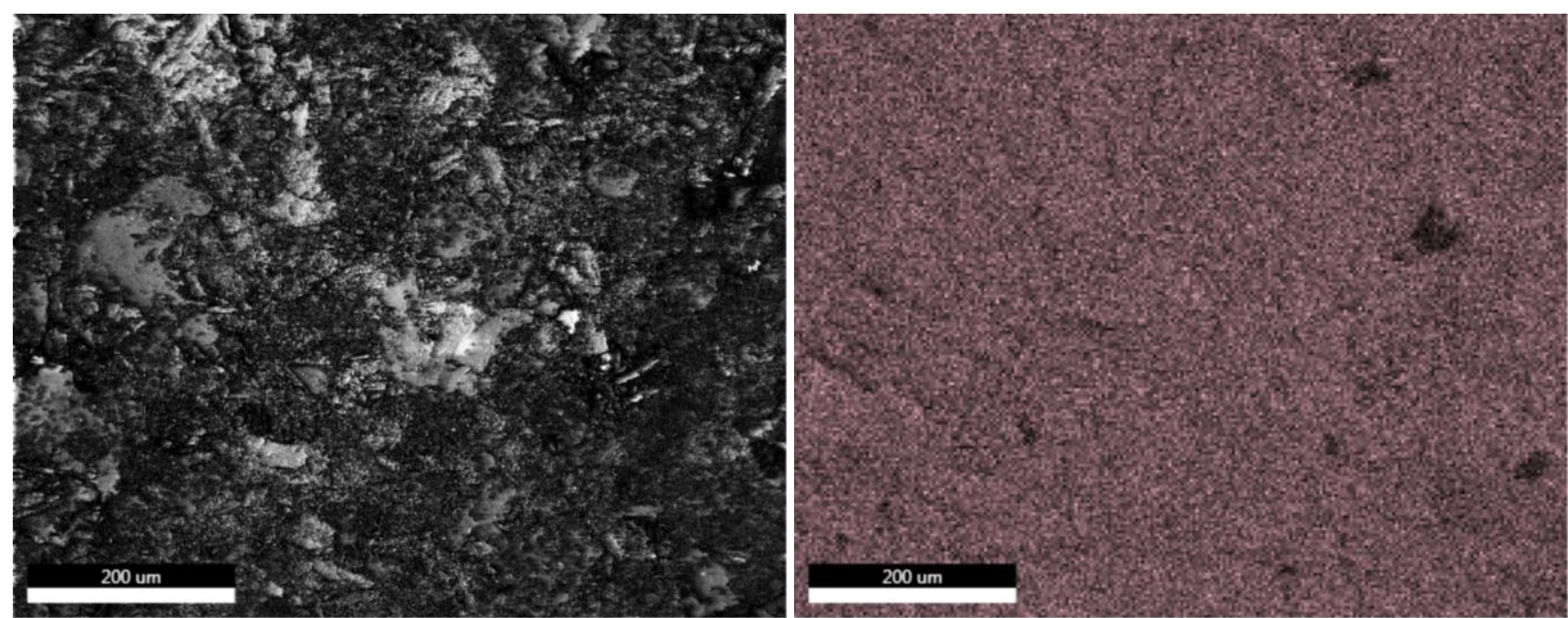

Figure 2. SEM image (left) and corresponding elemental map for nitrogen (beige) from the hot melt extrusion ASD model showing no differentiation between crystalline and amorphous APAP.

Disclosures:

All authors are employees of AbbVie and may own AbbVie stock. The design, study conduct, and financial support for this research were provided by AbbVie. AbbVie participated in the interpretation of data, review, and approval of the publication. 